\title{
多值逻辑函数相关免疫的谱特征 $*$
}

\author{
张木想 肖国镇 \\ (西安电子科技大学应用数学系, 西安 710071)
}

\section{关键词多值逻辑函数、相关免疫、Chrestenson 变换、纠错码}

相关免疫是密码学中的一个重要概念. 在文献[1] 中, Siegenthaler 给出了相关免疼的数学 定义, 并且将逻辑函数的相关免疫阶数作为密码系统抗相关攻击的一个度量指标. 利用 Walsh 变换, 文献[2] 研究了二值逻辑函数即布尔函数的相关免疫性, 得到了几变元的布尔函数为 $m$ 阶相关免疫的充分必要条件, 这一结果在研究二值相关免疫函数的性质及构造中发挥了重要 作用. 对于从 $G F^{n}(p)$ 到 $G F(p)$ 的函数, 即 $p$-值逻辑函数, 由于其代数结构比布尔函数复杂. 对 其相关免疫性的研究要比布尔函数困难得多. 用类似于文献[2] 的方法, 文献 [3] 用 Chrestenson 变换研究了 $p$-值逻辑函数的相关免疫性, 但只得到了 $n$ 变元的 $p$-值逻辑函数为 $m$ 阶相关免 疫的一个必要条件. 本文将一个 $p$-值逻辑函数变换为一个复值函数, 通过对该复值函数的 Chrestenson 变换的研究, 得到了 $n$ 变元的 $p$-值逻辑函数为 $m$ 阶相关免疫的充分必要条件.

设 $p$ 为素数, $\mathbb{Z}_{p}=\{0,1,2, \cdots, p-1\}$, 则 $\mathbb{Z}_{p}$ 在模 $p$ 加法和乘法运算下构成一个域, 且此域 与任何素域 $G F(p)$ 同构. 对任意 $X=\left(x_{1}, x_{2}, \cdots, x_{n}\right) \in \mathbb{Z}_{p}^{n}, X$ 中非零分量的个数称为 $X$ 的汉明重 量, 简记为 $W(X)$.

定义 1 设 $f$ 为 $\mathbb{Z}_{p}^{n}$ 到 $\mathbb{Z}_{p}$ 的函数, 对任意 $a \in \mathbb{Z}_{p}$, 若 $\left|\left\{X \mid X \in \mathbb{Z}_{p}^{n}, f(x)=a\right\}\right|=p^{n-1}$, 则称 $f$ 为平 衡的 $p$-值逻辑函数.

定义 2 设 $f$ 和 $g$ 为 $\mathbb{Z}_{p}^{n}$ 到 $\mathbb{Z}_{p}$ 的函数, $f$ 和 $g$ 的复合率定义为

$$
C(f, g)=\left|\left\{|X| X \in \mathbb{Z}_{p}^{n}, f(X)=g(X)\right\}\right| / p^{n} .
$$

定义 $3^{\text {|q }}$ 设 $f$ 为 $\mathbb{Z}_{p}^{n}$ 到 $\mathbb{Z}_{p}$ 的函数, $f$ 的 Chrestenson 变换为

$$
S_{f}(\omega)=p^{-n} \sum_{X \in \Sigma_{p}^{n}} f(X) u^{-\omega \cdot X},
$$

其中 $\omega \in \mathbb{Z}_{p}^{n}, u=e^{2 \pi i} / p, i=\sqrt{-1} \cdot \omega \cdot X$ 表示 $\omega$ 与 $X$ 的内积, 即 $\omega \cdot X=\omega_{1} x_{1}+\omega_{2} x_{2}+\cdots+\omega_{n} x_{n}$ $(\bmod p)$.

定义 4 设 $x_{1}, x_{2}, \cdots, x_{n}$ 为 $\mathbb{Z}_{p}$ 上独立同分布的随机变量, 并且对任意 $a \in \mathbb{Z}_{p}, p\left(x_{i}=a\right)=p^{-1}$, $1 \leqslant i \leqslant n$. 设 $f$ 为 $\mathbb{Z}_{p}^{n}$ 到 $\mathbb{Z}_{p}$ 的函数, $Z=f\left(x_{1}, x_{2}, \cdots, x_{n}\right)$. 如果对任意 $m$ 个变元 $x_{i_{1}}, x_{i_{2}}, \cdots, x_{i_{m}}$, 随 机变量 $Z$ 与 $x_{i_{1}}, x_{i_{2}}, \cdots, x_{i_{m}}$ 统计独立, 则称 $f$ 为 $m$ 阶相关免疫函数.

若将 $\mathbb{Z}_{p}^{n}$ 看成是 $\mathbb{Z}_{p}$ 上的 $n$ 维线性空间, 对于 $\mathbb{Z}_{p}^{n}$ 的线性子空间 $V, V$ 的对偶空间定义为

$$
V^{\perp}=\left\{X \mid X \in \mathbb{Z}_{p}^{n}, X \cdot Y=0, \otimes Y \in \mathbb{Z}_{p}^{n}\right\} .
$$

1993-07-12 收稿, 199401-14 收修改稿.

*国家自然科学基金和国家教委博士点研究基金资助项目. 
引理 1 设 $V$ 是 $\mathbb{Z}_{p}^{n}$ 的 $k$ 维子空间, 则

$$
\sum_{X \in V} u^{\omega \cdot X}= \begin{cases}p^{k} & \omega \in V^{\perp}, \\ 0 & \omega \notin V^{\perp} .\end{cases}
$$

引理 2 设 $a_{0}, a_{1}, \cdots, a_{p-1}$ 为有理数, 则 $\sum_{k=0}^{p-1} a_{k} u^{k}=0$ 当且仅当 $a_{0}=a_{1}=\cdots=a_{p-1}$.

设 $f$ 为 $\mathbb{Z}_{p}^{n}$ 到 $\mathbb{Z}_{p}$ 的函数. 对任意 $X \in \mathbb{Z}_{p}^{n}$, 设 $(f)(x)=u^{f(x)}$, 则 $(f)$ 为 $\mathbb{Z}_{p}^{n}$ 到复数域 $\subseteq$ 的函数, $(f)$ 的 Chrestenson 变换为

$$
S_{(f)}(\omega)=p^{-n} \sum_{x \in \mathbb{Z}_{p}^{*}} u^{f(x)-\omega \cdot X} .
$$

基于引理 1 和引理 2, 可以证明如下的定理:

定理 1 设 $f$ 为 $\mathbb{Z}_{p}^{n}$ 到 $\mathbb{Z}_{p}$ 的函数, 则 $f$ 为 $m$ 阶相关免疫的充分必要条件是, 对任意 $\omega \in \mathbb{Z}_{p}^{n}$, $0<W(\omega) \leqslant m, S_{(f)}(\omega)=0$.

设 $L_{\omega, r}(X)=\omega \cdot X+r$ 为线性函数, 由定理 1 和引理 2 容易证明: $f$ 为 $m$ 阶相关免疫函数 的充分必要条件是, 对任意 $\omega \in \mathbb{Z}_{p}^{n}, c<W(\omega) \leqslant m$, 以及任意 $r \in \mathbb{Z}_{p}, C\left(f, L_{\omega, r}\right)=1 / p$.

定理 2 设 $f\left(x_{1}, x_{2}, \cdots, x_{n}\right)$ 为平衡的 $p$-值逻辑函数, 则 $f$ 为 $m$ 阶相关免疫的充分必要条 件是, 对任意 $m$ 个变元 $x_{i_{1}}, x_{i_{2}}, \cdots, x_{i_{m}}$ 以及这 $m$ 个变元的任意 $p$-值逻辑函数 $g\left(x_{i_{1}}, x_{i_{2}}, \cdots, x_{i_{m}}\right)$, $C(f, g)=1 / p$.

定理 3 设 $V$ 为 $\mathbb{Z}_{p}$ 上的 $[n, k]$ 线性分组码, 其最小距离和生成矩阵分别为 $d$ 和 $G$. 设 $g^{\prime}$ 为 $\mathbb{Z}_{p}^{n}$ 到 $\mathbb{Z}_{p}$ 的函数. 对任意 $X \in \mathbb{Z}_{p}^{n}$, 设 $f(x)=g\left(X \cdot G^{t}\right)$, 则 $f$ 为 $d-1$ 阶相关免疫函数.

定理 3 表明, 利用 $\mathbb{Z}_{p}$ 上的线性分组码可以构造出 $p$-值相关免疫函数, 并且码的最小距离 越大, 逻辑函数的相关免疫阶数越高. 特别是, 当 $g$ 为 $\mathbb{Z}_{p}$ 到 $\mathbb{Z}_{p}$ 的函数时, $f\left(x_{1}, x_{2}, \cdots, x_{n}\right)=g\left(x_{1}+\right.$ $\left.x_{2}+\cdots+x_{n}\right)$ 为 $n-1$ 阶相关免疫函数. 因此, $p>2$ 时, 具有最高相关免疫阶数的 $p$-值逻辑函数 可以不是线性函数. 这与具有最高相关免疫阶数的布尔函数必是线性函数有较大差别. 如何 构造具有最高相关免疫阶数同时又有最高非线性阶数的 $p$-值逻辑函数, 是一个值得进一步研 究的课题.

\section{参考文裁}

[1] Siegenthaler, T., IEEE Trans. Infor. Theory, 1984, IT-30(5): 776-780.

[2] Xiao Guo-zhen, Massey, J. L., IEEE Trans. Infor. Theory, 1988, IT-34(3): 569-571.

[3] 武传坤, 电子科学学刊, 1993, 15(1): 17-25.

[4] Karpousky, M. G., Finite Orthogonal Series in the Design of Digital Devices, Wiley and IUP, New York, Jerusalem, 1976. 